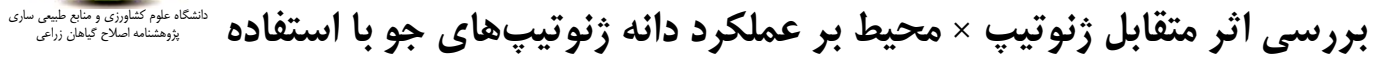

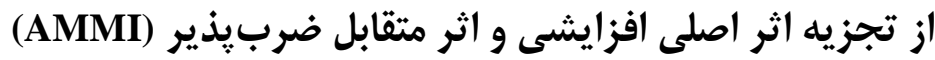

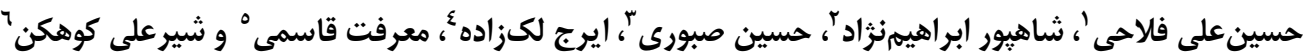

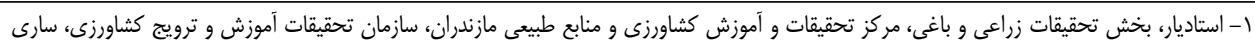

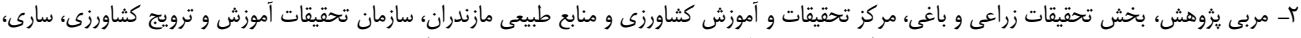

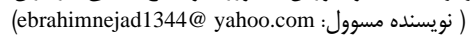

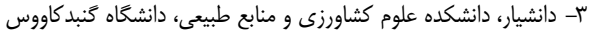

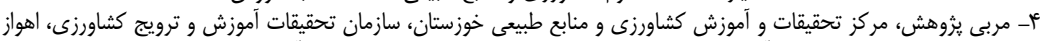

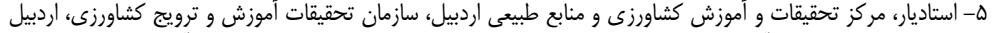

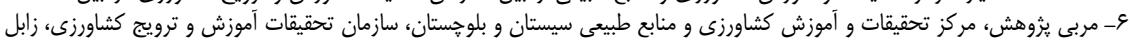

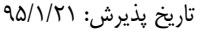
تاريخ دريافت:

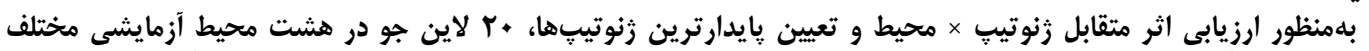

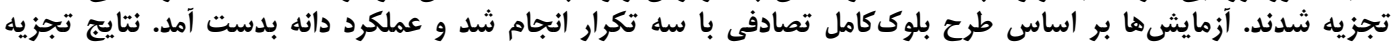

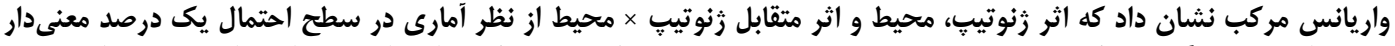

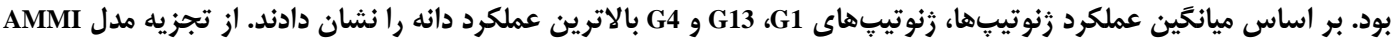

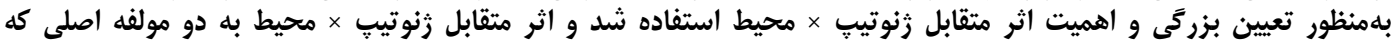

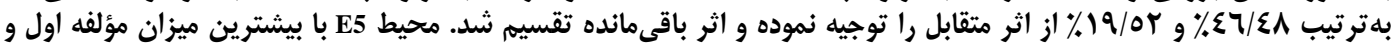

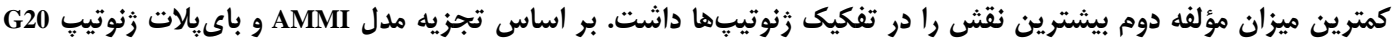

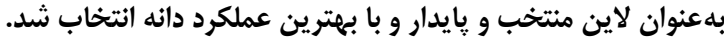

وازههاى كليدى: يايدارى، تجزيه AMMI، جو، عملكرد دانه

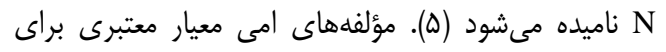

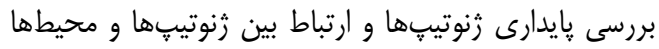

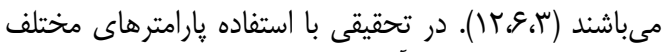

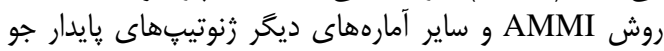

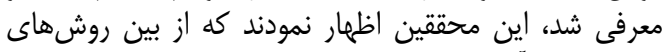

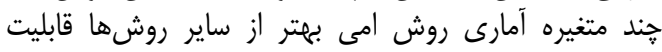

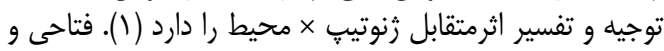

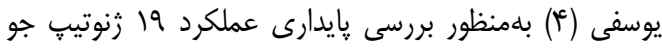

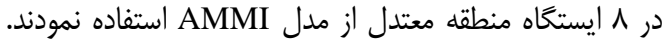

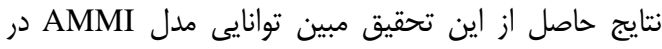

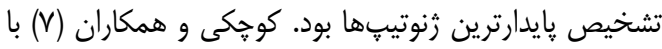

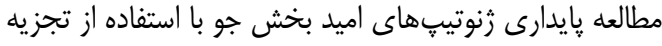

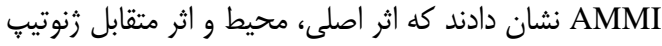

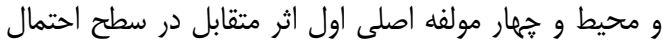
يك درصد معنى دار بودند.

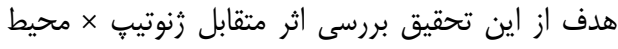

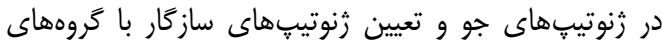
محيطى و شناسايى ارقام يايدار بود.

\section{مواد و روشها}

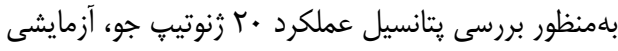

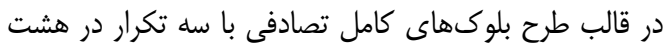

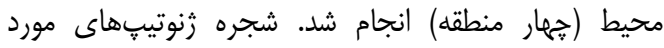

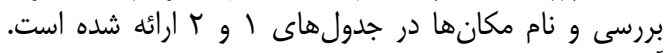

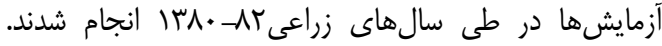

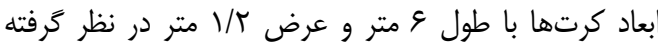

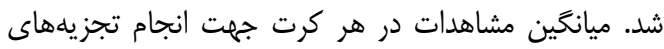

مقدمه مهرم مىباشد كه علاوه بر مصارف خوراكى داراى مصارف

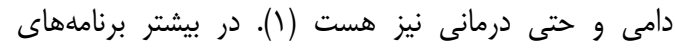

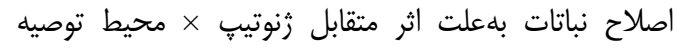

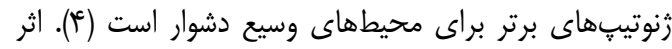

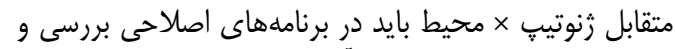

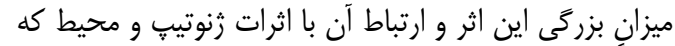

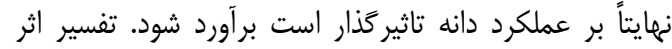

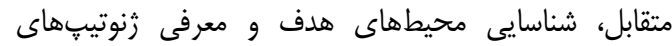

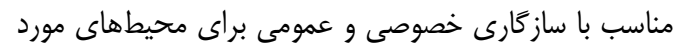

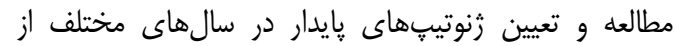

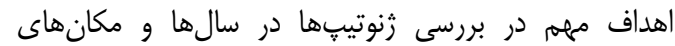

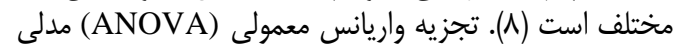

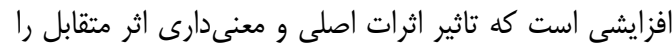

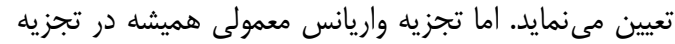

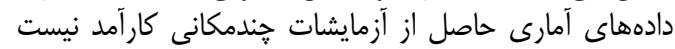

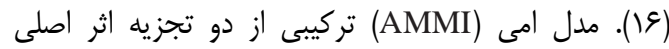

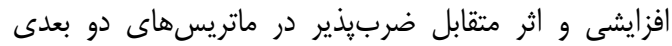

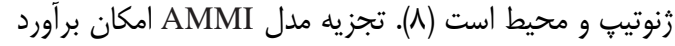

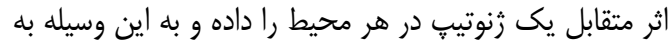

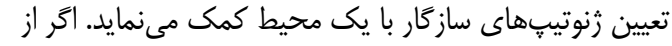

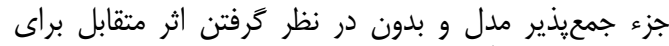

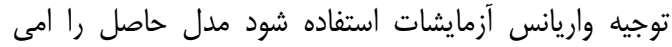

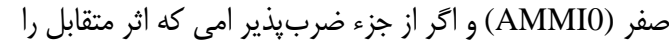

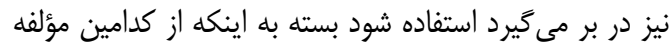
امى استفاده شود، مدل امى التا امى ( 


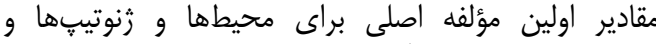

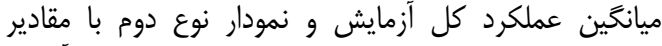

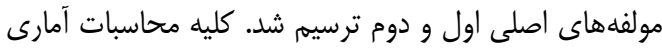

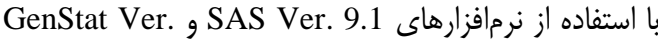
9.2

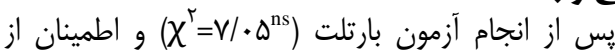

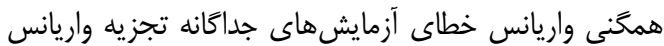

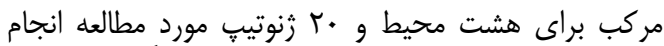

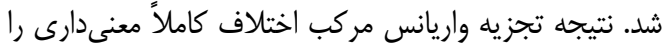

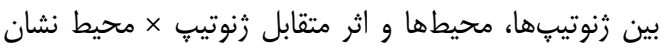

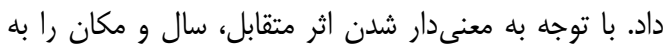

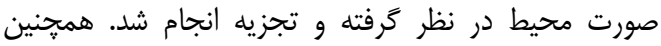

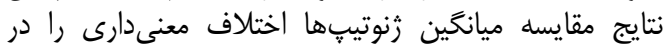

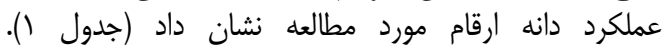

آمارى مورد استفاده قرار گرفت. در ابتدا جهت بروسى اوليه

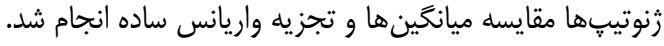

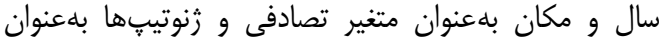

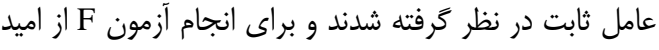

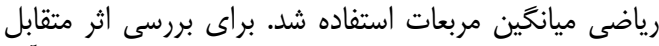

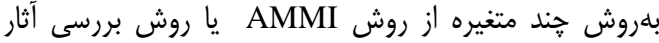

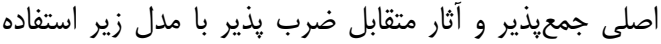
$\mathrm{Y}_{\mathrm{ij}}=\mu+\mathrm{g}_{\mathrm{i}}+\mathrm{e}_{\mathrm{j}}+\sum_{\mathrm{k}=1}^{\mathrm{N}} \lambda_{\mathrm{k}} \alpha_{\mathrm{ik}} \gamma_{\mathrm{jk}}+\varepsilon_{\mathrm{ij}}$

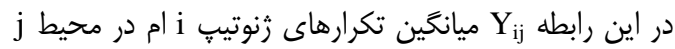

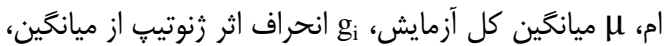

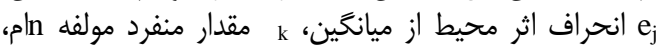

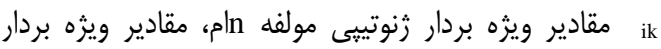

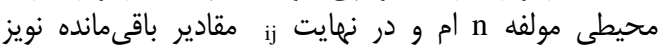

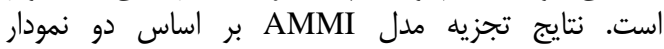
باىيلات تجزيه AMMI تفسير شد. نمودار نوع اول بر اساس دول ندار

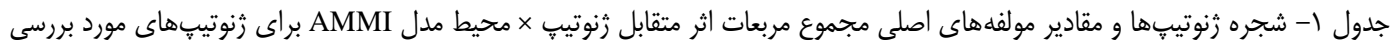
Table 1. Pedigree of genotypes and principle component of sum of square genotype by environment interaction for AMMI model in evaluated genotypes

\begin{tabular}{|c|c|c|c|c|c|}
\hline IPCA 2 & IPCA 1 & (تن در هكتار) & رتبه & شجره & اختصارى علامت \\
\hline$-\cdot /$ DTF & $\cdot /$ / q & $\varphi / \mu^{a}$ & 1 & Check-1(Sahra) & G1 \\
\hline$\cdot 1 \cdot+q$ &.$/ \mathrm{V} \Delta \Delta$ & $\Gamma / \Delta q^{c}$ & r. & NKERR/ARG8/6/UC566/5/M64-76/BON//JO/YORK/3/M5/ & G2 \\
\hline r & $\cdot / / \omega$ & $r / T r^{\mathrm{abc}}$ & V & ARIZONA5908/ATHS//LIGNEE640/6/LIGNEE527/5/AS54 & G3 \\
\hline.$/ 014$ & $-\cdot / \Delta$ & $f / r \Delta^{\mathrm{ab}}$ & r & ARIZONA5908/ATHS//LIGNEE640/6/LIGNEE527/5/AS54/ & G4 \\
\hline$\cdot / \backslash \wedge \Delta$ & $-\operatorname{thet}$ & $\tau / r \Lambda^{a b c}$ & f & ARIZONA5908/ATHS//LIGNEE640/6/LIGNEE527/5/AS54/ & G5 \\
\hline.$- \cdot 1 \cdot r \Lambda$ &.$/$ fre & $f / \cdot$ fabc $^{2}$ & it & ARIZONA5908/ATHS//LIGNEE640/6/LIGNEE527/5/AS54/ & G6 \\
\hline$-\cdot / 8$ & 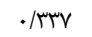 & $\mathrm{r} / \mathrm{V}^{\mathrm{abc}}$ & iV & LIGNEE527/5/AS54/TRA//CER*2/TOLI/3/AVT/TOLI//BZ & G7 \\
\hline.$/ 4 \& \Delta$ &.$- \cdot 1 \cdot v 9$ & ५/q rabc & 10 & HARI/NOPAL-CMSW & G8 \\
\hline ./rq &.$/ \cdot r \mathrm{~V}$ & $\Gamma / \Lambda \Delta^{\mathrm{abc}}$ & 19 & "VIRINGA"S"/BOLDO"S & G9 \\
\hline$-\bullet / \& \wedge \Delta$ &.$/ \mathrm{FV}$ & $\varphi / Y^{a b c}$ & $\varepsilon$ & IRANSHAHR & G10 \\
\hline . &.$- /$ Tf & $\varphi / \cdot \Delta^{\text {abc }}$ & 11 & ARAR/APM & G11 \\
\hline$-\cdot / 4 T h$ & $\cdot 1 \cdot \mathrm{V}$ & $r / \cdot v^{a b c}$ & 1. & POAL//QUINA & G12 \\
\hline$\cdot / V^{e}$ &.$/ 091$ & $F / r q^{a b}$ & r & CIN-B/80.5138//BLORIA-BAR/EOPAL3 & G13 \\
\hline$\cdot / t+t$ &.$- / N \& t$ & ५/৭qabc & سו & ../ABET//GLORIA-BAR/COME-B/3/SEN/4 & G14 \\
\hline$\cdot / T$ & 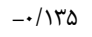 & $r / /^{\mathrm{abc}}$ & $\wedge$ & WI229//MZQ//DL7/3/WI2198/EMIR & G15 \\
\hline.$- / 1 F \Delta$ &.$/ 948$ & T/q̆c & 19 & L. $131 /$ Trean 78 & G16 \\
\hline.$- / 1 Q T$ & - & $\mathrm{m} / \mathrm{Q}^{\mathrm{abc}}$ & if & L.527//Bahtim/P171...xBiran/Una 8271 & G17 \\
\hline TET & $-* / F \Delta \Delta$ & $\varphi / \cdot V^{\mathrm{abc}}$ & 9 & Rihane/Research & G18 \\
\hline$-\cdot / v \cdot r$ & $-\cdot / \Lambda \cdot \Lambda$ & $r / q \Lambda^{\mathrm{bc}}$ & M & PMb-12 & G19 \\
\hline$-\cdot / \mathrm{TQ \Lambda}$ & $-* / 4 \mid \Lambda$ & $\tau / r V^{a b c}$ & $\Delta$ & WB-73-6 & $\mathrm{G} 20$ \\
\hline
\end{tabular}

Means with similar letters in each column are not significantly different at 5\% probability level (DMRT).

G15 بلترتيب كمترين مقادير را براى مولفه اول (IPCA1)

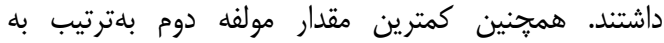

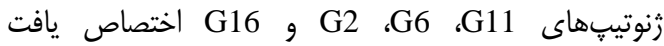

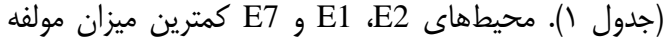

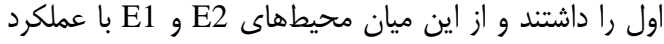

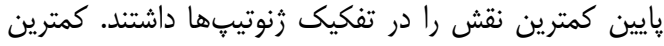

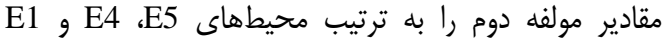

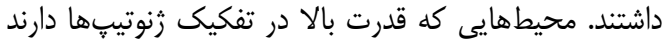

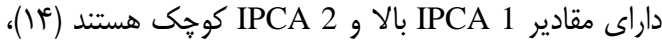
بر اين اساس محيط E5 با بيشترين ميزان مولفه اول و
زنوتيت G1 و G2 بلترتيب با ميانخين عملكرد رس/ أ و

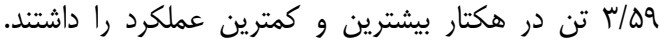

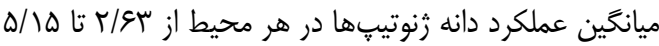

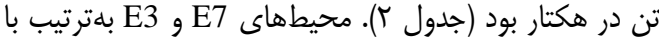

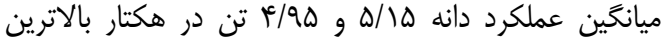

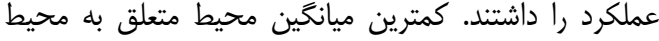

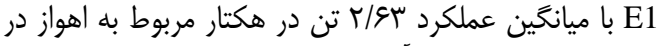

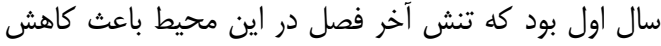

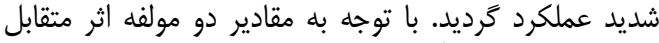

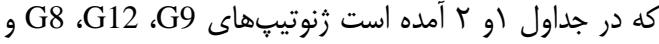




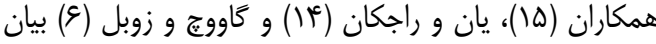

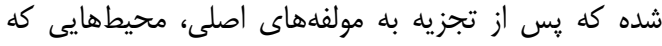

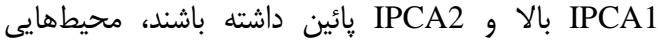
هستند كه قدرت تمايز حنين زنوتيبهايى را دارند.

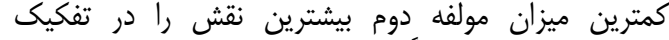

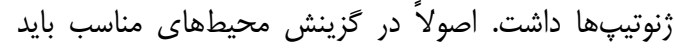

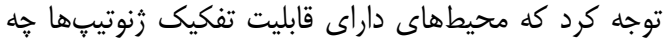

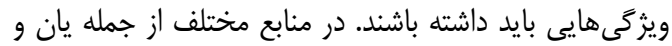

جدول r- مقادير مولفههاى اصلى در مجموع مربعات اثر متقابل زنوتيّ × محيط در مدل AMMI براى محيطهاى مورد بررسى Table 2. Principle component of sum of square genotype by environment interaction for AMMI model in evaluated

\begin{tabular}{|c|c|c|c|c|c|}
\hline IPCA 2 & IPCA 1 & ميانكَين & رتبه & محيط & علامت اختصارى \\
\hline$-0 / T K F$ & $\cdot 1 \cdot 11$ & $r / q^{\mu e}$ & $\wedge$ & اهواز سال اول & E1 \\
\hline$-/ \pi \Delta \Delta$ & $.1 . .9$ & $F / T q^{b c}$ & i & اهواز سال دوم & E2 \\
\hline I/TAT & .10 .9 & $r / 9 \Delta^{a b}$ & r & زابل سال اول & E3 \\
\hline . /NE & $-1 / 991$ & $\Gamma / \Lambda^{c d}$ & 9 & زابل سال دوم & E4 \\
\hline.$/ 11 f$ & /DFS & $\Gamma / \Lambda Q^{\mathrm{ed}}$ & v & مغان سال اول & E5 \\
\hline 年/ & . ITM & $\mathrm{c} / \mathrm{rbc}^{\mathrm{bc}}$ & r & مغان سال دوم & E6 \\
\hline ג ג וק/.- &.$|r \||$ & $\Delta / / \Delta^{a}$ & 1 & كنبد سال اول & E7 \\
\hline$-|N| \mid$ & הFA & $r / r^{c}$ & $\Delta$ & كنبد سال دوم & E8 \\
\hline
\end{tabular}

ثنوتيڤ × محيط را توجيه نمود. بزرگى اثر محيط بيانگر تفاوت

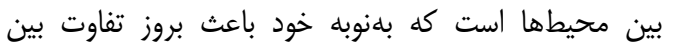

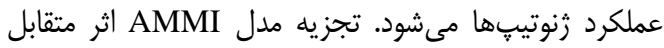

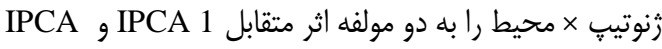

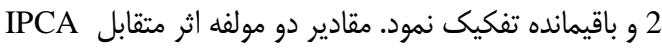
1

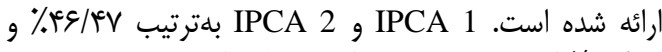

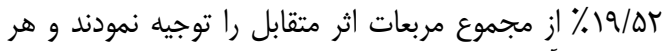

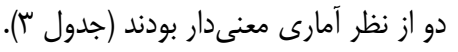

تجزيه مدل AMMI تهز مدا AMI

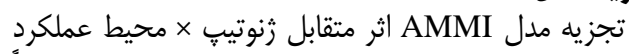

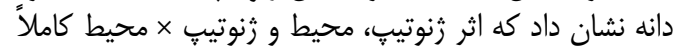

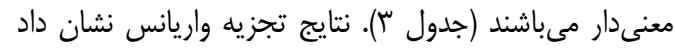

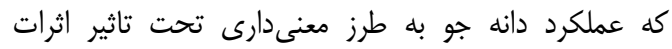

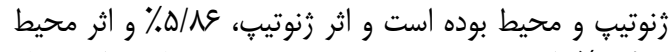

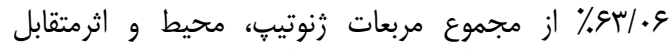

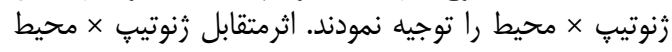

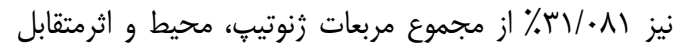

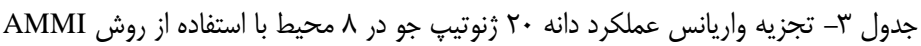
Table 3. Analysis of variance for 20 barley genotypes in 8 environment using AMMI methods

\begin{tabular}{|c|c|c|c|c|c|}
\hline درصد مجموع مربعات اثرمتقابل زنوتيب × & $\mathrm{F}$ & ميانكين مربعات & مجموع مربعات & درجه أزادى & منابع تغيير \\
\hline & $\left.N / f^{4}\right) * *$ & $r / \cdot I V$ & $\mathrm{fVq} / \mathrm{V}$ & 109 & تيمار \\
\hline & $r / / r^{* * *}$ & $1 / 4 \mathrm{~V} \Lambda$ & $r N / 1$ & 19 & رُنوتي \\
\hline & 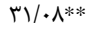 & FT/TIV & $r \cdot T / \Delta$ & v & محيط \\
\hline & $r / / r^{* *}$ & $|/| r \mid$ & $1 F q / 1$ & אזו & رنوتيِ × محيط \\
\hline$F q / F V$ & $V / V T^{* * *}$ & $r / W V$ & $99 / \pi$ & rQ & IPCA1 \\
\hline $19 / 01$ & $r / \Delta \Gamma^{* * *}$ & $1 /$ TAF & $r q / 1$ & זr & IPCA2 \\
\hline$r \varphi / \cdot V$ & $1 / 99 * *$ & .1091 & $0 \cdot / V$ & $\Lambda \Delta$ & باقيمانده \\
\hline & &.$/ 409$ & $1.9 / 1$ & $r \cdot \varphi^{2}$ & |شتباه \\
\hline
\end{tabular}

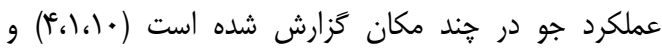

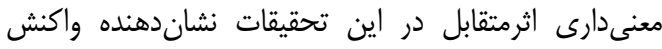

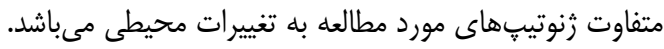

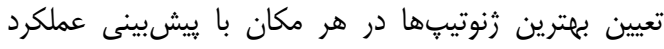

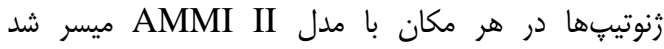

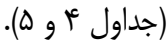

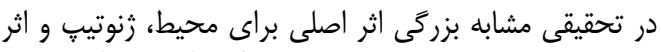

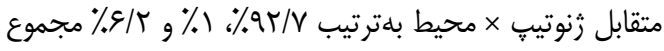

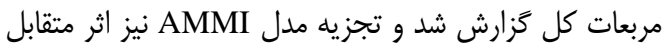

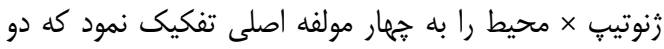

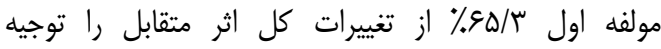

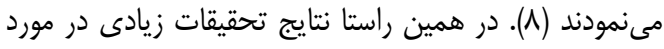

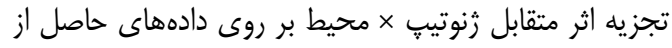


جدول F- مقادير برآوردى مدل AMMI از عملكرد زنوتيّها (تن در هكتار) در محيطهاى مورد بررسى

\begin{tabular}{|c|c|c|c|c|c|c|c|c|}
\hline محيط 1 & محيط V & محيط 9 & ه ه ه D & محيط f & محيط r & محيط r & محيط 1 & علامت اختصارى \\
\hline$r / q \Delta q$ & Q/9IV & $\digamma / \Delta Q f^{F}$ & $r / q \times q$ & $r / T \Delta V$ & F/ATF & $F / V V \Delta$ & $r / T r V$ & G1 \\
\hline$r / \Lambda \cdot{ }^{+}$ & $F / \lambda F$ & $4 / .91$ & אזس// & I/vag & F/৭६ & $r / \Lambda r q$ & r/rrq & G2 \\
\hline $4 / \cdot 1$ & D/IG & $F / 9 Q 1$ & $\Gamma / \Delta \cdot 1$ & r/rat & D/gFt & F/rEV & T/VTI & G3 \\
\hline r/VIT & س & $r / 999$ & אוז/א & $\Delta / T V V$ & Q/दQT & $F / \& V \Delta$ & $r / V \wedge D$ & G4 \\
\hline $5 / .19$ & D/TM & $F / \Delta F \Delta$ & T/TVD & F/DGT & $\Delta / \mu \cdot \Gamma$ & F/FAT & $r / \Lambda \cdot 9$ & G5 \\
\hline T/TIT & $\Delta / T V \Psi$ & F/4Fq & $r / 4 \cdot \Delta$ & r/AFA & $\Delta / / \Delta S$ & $\varphi / \mu \cdot \omega$ & $r / 9 \vee q$ & G6 \\
\hline$r / \pi \cdot 9$ & D/R\&I & r/Als & T/QIV & $r / 9 V T$ & $r / . . V$ & $r / 1 \cdot r$ & $r / 4+\Lambda$ & G7 \\
\hline$r / F V T$ & $r / M r$ & F/MEY & عץ. & r/QY^ & $\Delta / r \wedge \Delta$ & $\varphi / \Delta \mu$ & r/ral & G8 \\
\hline$r / \Delta \cdot \Lambda$ & $r / V T \Delta$ & $F / T N E$ & $r / \mu$ & $r / g r$ & $\Delta / r 9 \Delta$ & $f / \cdots 1$ & | & G9 \\
\hline$r / 994$ & D/998 & F/TQ & $r / r V$ & $r / q r$ & $r / 9 \cdot \mu$ & $4 / 919$ & $r / 9 \Delta \mu$ & G10 \\
\hline r/qYq & $0 / .91$ & $F / T \Delta D$ & $r / \mu$ & $r / r+1$ & r/AVT & f/raq & $r / 9 / f$ & G11 \\
\hline Ther & $\Delta / \tilde{A V}$ & $r / T \Lambda r$ & $r / 199$ & r/gIr & $\Gamma / \notin 9 D$ & 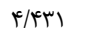 & $r / V 91$ & G12 \\
\hline r/११९ & كسות & ه/.91 & r/MR & $r / \cdot v 1$ & G/DTE & אוז/F & $r / \Lambda F 1$ & G13 \\
\hline$r / \Delta \cdot r$ & $r / v 9 q$ & $4 / \cdot 19$ & $r / 9 \Delta \Delta$ & Q/QT & F/VYG & $4 / 1 \wedge q$ & $r / \notin 94$ & G14 \\
\hline$r / \Lambda \Delta S$ & $\Delta / \cdot \Delta T$ & r/rqv & $r /|Q|$ & $F /$ ISq & ه/।qT & $f / r 9 \Delta$ & r/GYA & G15 \\
\hline $4 / .9$ & ( & $r / l \cdot r$ & T/TTV & $1 / 019$ & r/Ars & r/qrq & 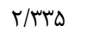 & G16 \\
\hline r/q४q & سזו/D & $r / \cdot \wedge V$ & T/MF & F/ETD & F/OTr & $F / T V D$ & r/QVq & G17 \\
\hline$r / 9 \wedge F$ & F/qur & $r / r q \Lambda$ & $r / ৭ \Delta \wedge$ & $f /$ r८q & D/.GT & $F / T \Delta \Lambda$ & r/DSG & G18 \\
\hline 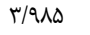 & $\Delta / \cdot V I$ & $r / 4 r q$ & T/TAT & F/QTV & $r / T V \mu$ & $F / 1.4$ & $\Gamma / \Gamma \Delta \Delta$ & G19 \\
\hline אוץא/F & $\Delta / \& \& D$ & $F / \widetilde{\mu} / f$ & $\Gamma / I T \Delta$ & F/ATD & $r / q 41$ & $r / 09$ & $r / M G$ & G20 \\
\hline
\end{tabular}

Tables5. Four primary selective of AMMI for per environment

جدول ه- جِهار انتخاب اول AMMI براى هر محيط

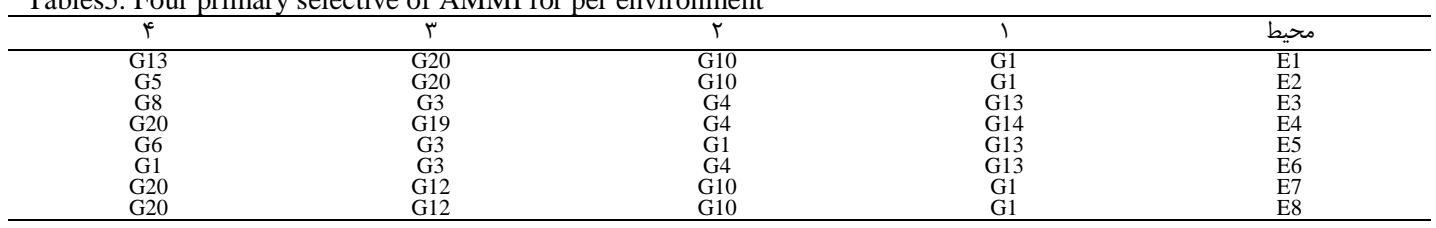

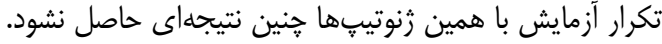

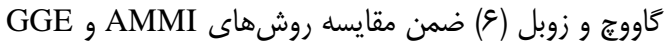

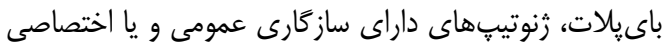

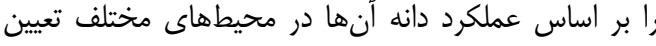

تجزيه باى يلات تجرد

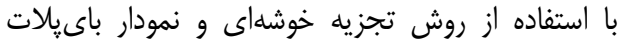

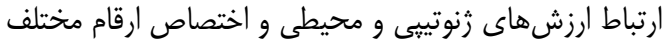

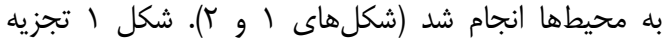

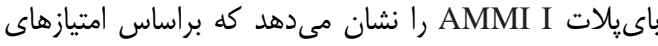

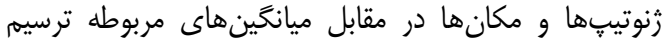

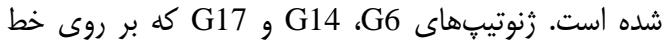

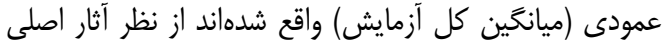

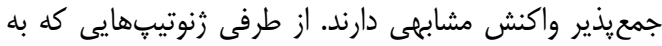

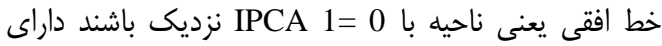

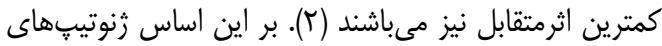

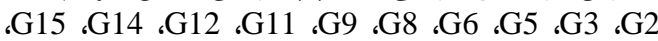

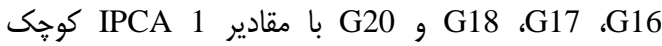

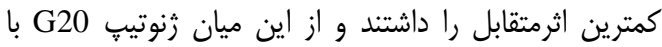

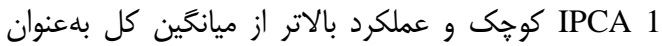

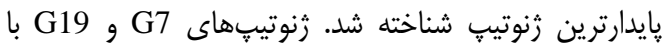

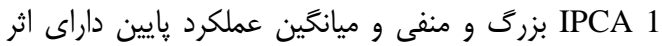

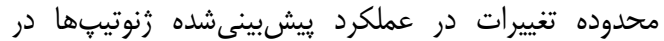

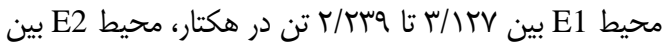
F/VVD

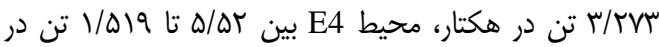
هكتار، محيط E5 بين

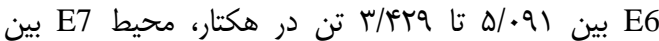

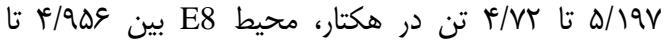
يُش

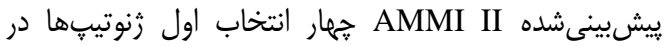

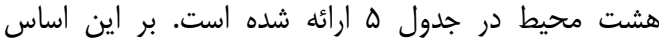

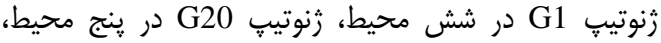

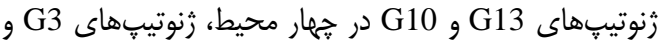

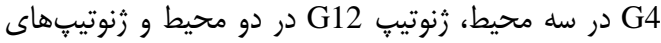

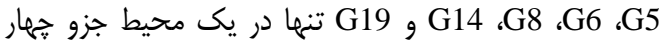

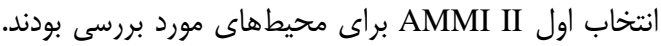

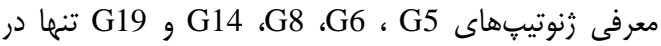

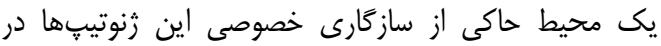

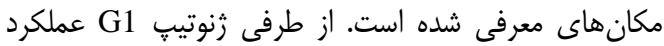

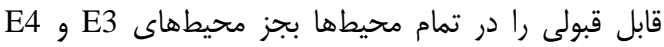

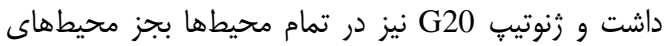
E3

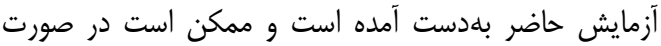




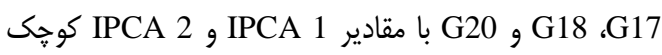
نزديك به محور مختصات قرار داشتند و كمترين اثرمتقابل

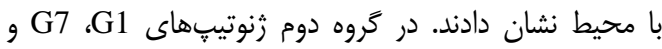

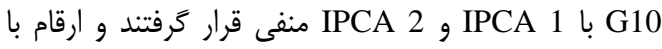

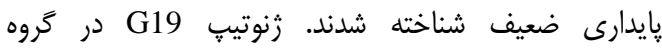

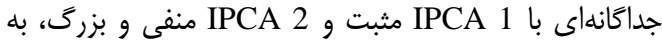

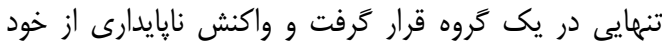

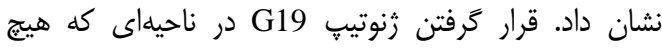

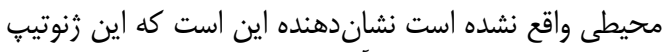

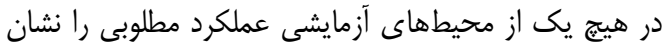

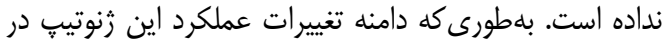

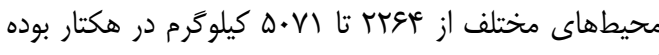

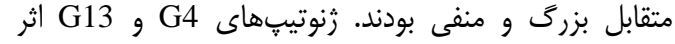

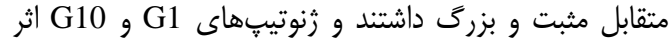

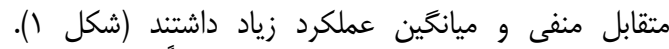

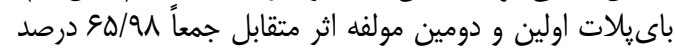

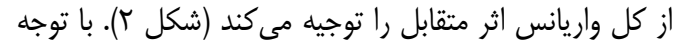

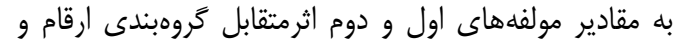

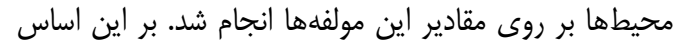

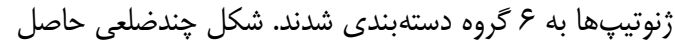

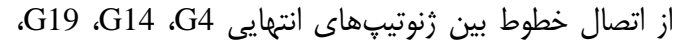

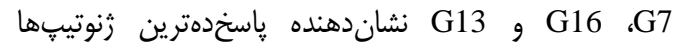

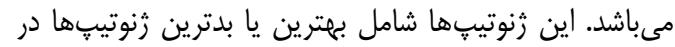

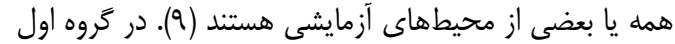

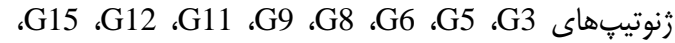

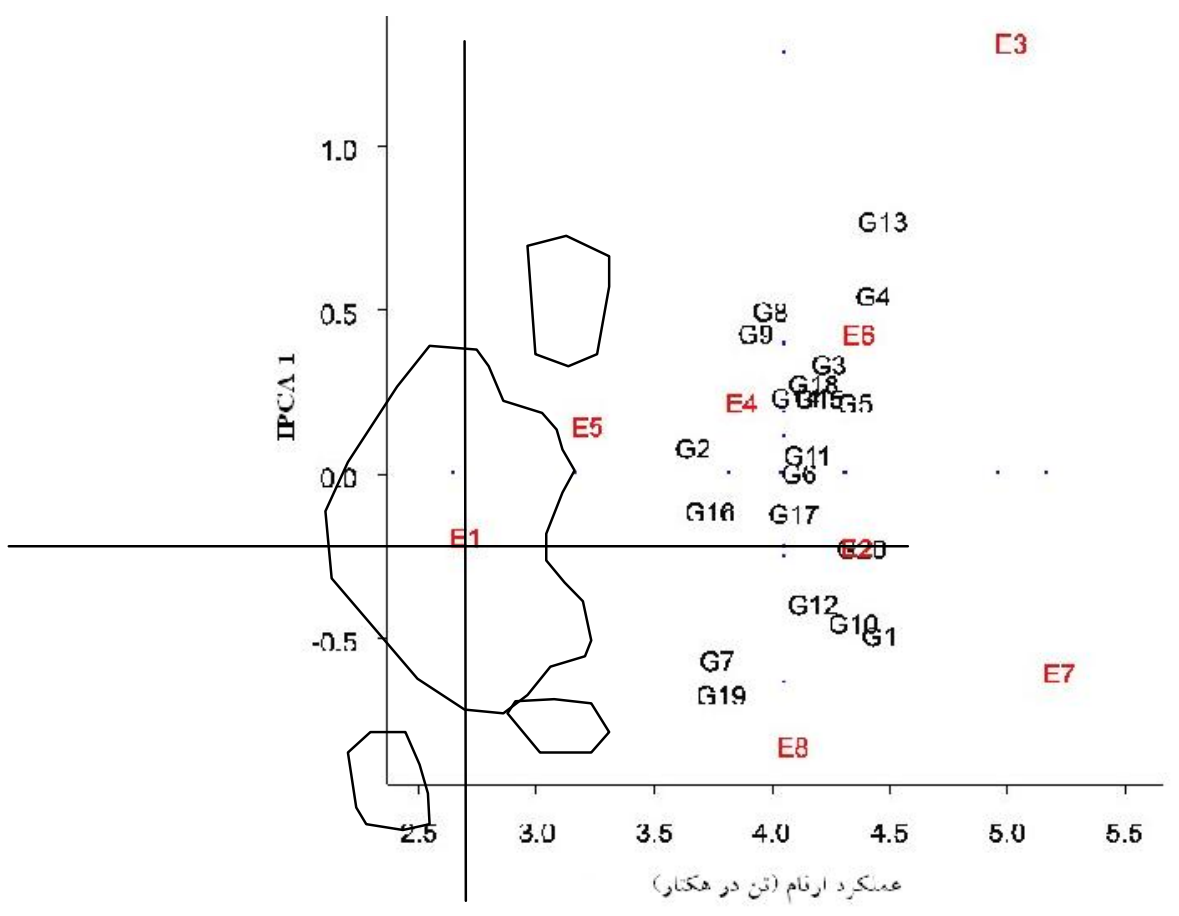

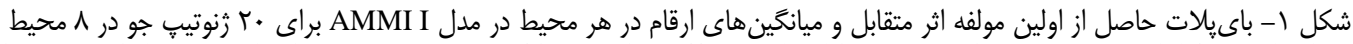
Figure 1. Biplot caused primery component of interaction effect and cultivar means in AMMI I model for 20 barley genotypes in 8 environment 


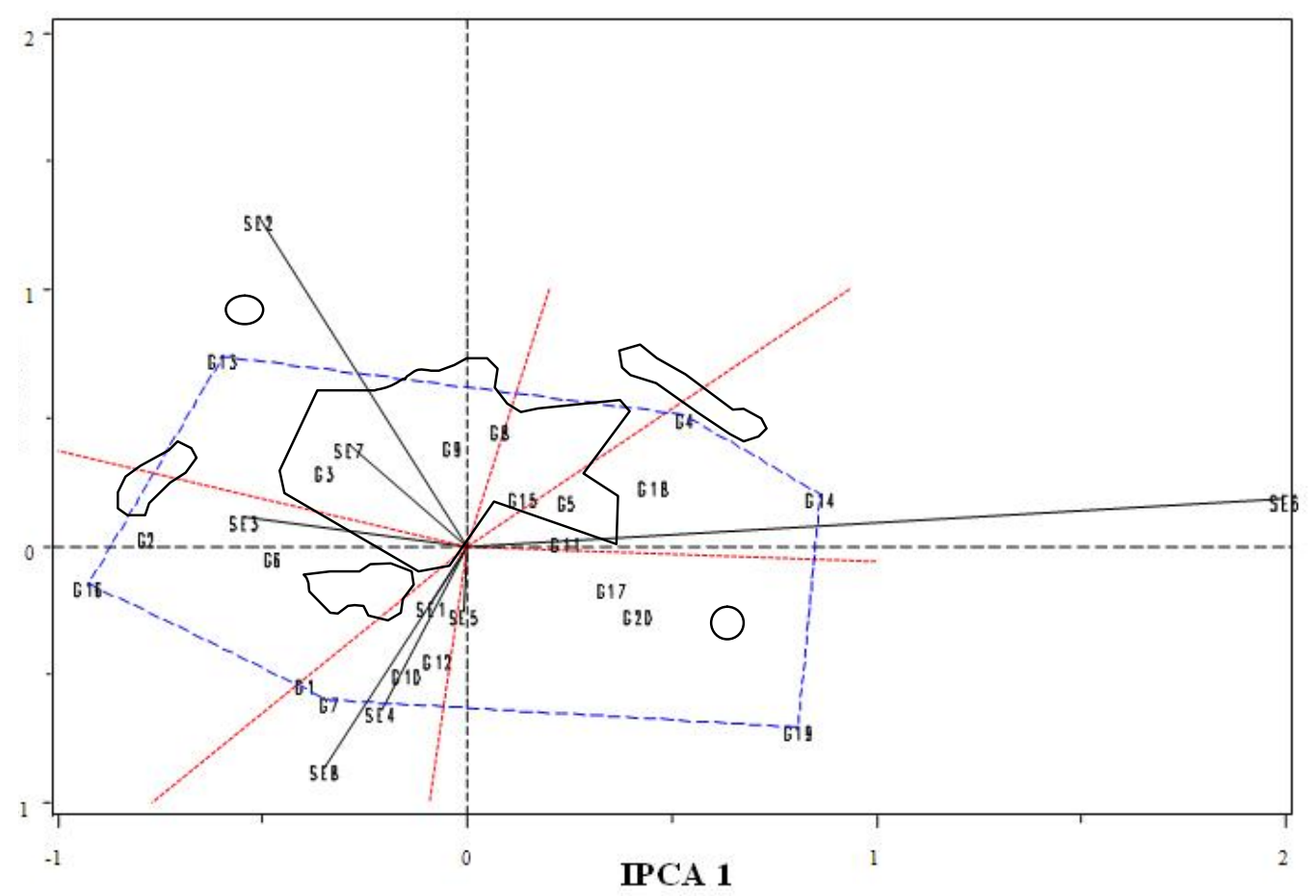

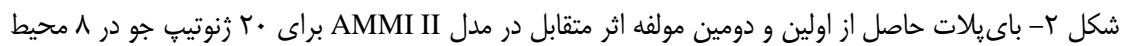
Table 2. Biplot caused primery and secondry component of interaction effect for AMMI II in 20 barley genotypes and 8 environments

همبستخى بالايى با يكديگر داشته و نقش مشابهى در زَزينش

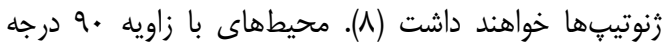

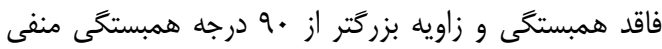

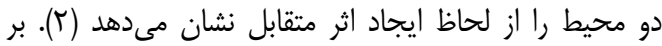

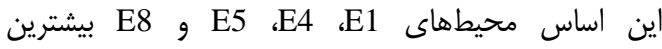

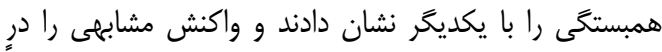

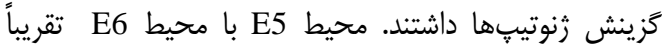

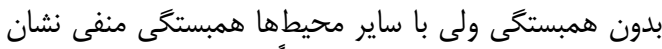

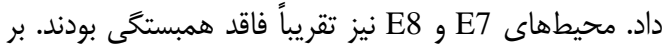

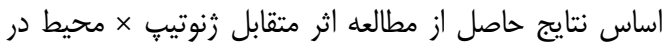

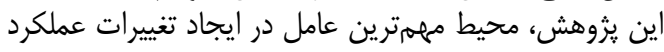

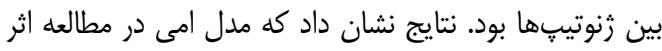

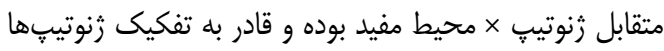

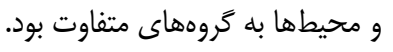

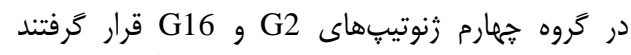

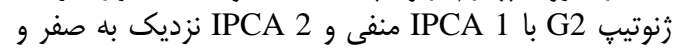

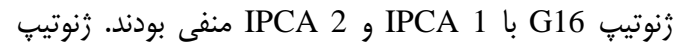

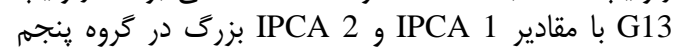

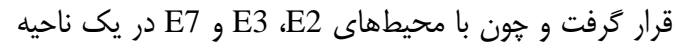

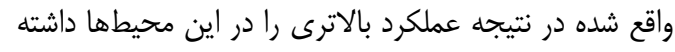

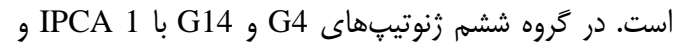

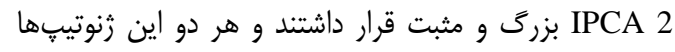

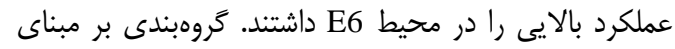

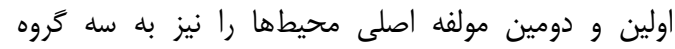

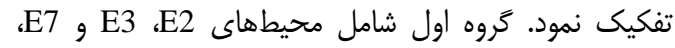

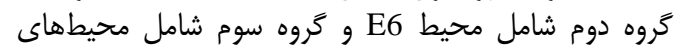

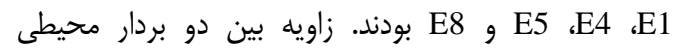

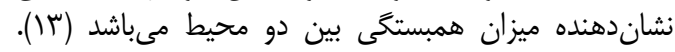

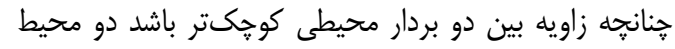


1. Ali Akbarpour, O., H. Dehghani and B. Sorkhi laleh lou. 2011. Investigating univariate and multivariate stability parameters of barley (Hordeum voulgare L.) promising genotypes in cold climate of Iran. Iranian Journal of Field Crop Science, 42: 23-32 (In Persian).

2. Askarinia, P., G. Saeidi and A. Rezai. 2009. Pattern analysis of genotype $\times$ field environments interaction for grain yield in wheat using AMMI method. Electronic Journal of Crop Production, 2: 75-90 (In Persian).

3. Das, S., R.C. Misra, M.C. Patnaik and S.R. Das. 2010. GxE interaction, adaptability and yield stability of mid-early rice genotypes. Indian Journal of Agricultural Research, 44: 104-111.

4. Fattahi, F. and A. Yousefi. 2006. The assessment of stability of barely yield genotypes (Hordeum vulgare L.) using replicable stability parameters and pattern analysis with AMMI model. Iranian Journal of Agriculture Science, 37: 317-326 (In Persian).

5. Gauch, H.G. and R.W. Zobel. 1996. AMMI analysis of yield trials. In: Kang, M. S. and H. G. Jr. Gauch (eds), Genotype- by -environment interaction. (pp: 85-122). CRC Press, Boca Raton, Florida, 344 pp.

6. Gauch, H.G. and R.W. Zobel. 1997. Identifying mega-environments and targeting genotypes. Crop Science, 37: 311-326.

7. Koocheki, A.R., B. Sorkhi and M.R. Eslamzadeh Hesari. 2013. Study on Stability of Elite Barley (Hordeum vulgare L.) Genotypes for Cold Regions of Iran Using AMMI Method. Cereal Research, 2 : 249-261.

8. Mohammadi, R., M. Armivan and M.M. Ahmadi. 2011. Genotype $\times$ Environment Interactions for Grain Yield of Durum Wheat Genotypes Using AMMI Model. Seed and Plant Improvement Journal, 27: 183-198 (In Persian).

9. Mostafavi, K., S. Hosseini Imeni and M. Zare. 2010. Stability Analysis of Rice Genotypes Based GGE biplot Method in North of Iran. Journal of Applied Sciences Research, 7: 1690-1694 (In Persian).

10. Nikkhah, H.R., A. Yousefi, S.M. Mortazavian and M. Arazmjoo. 2007. Analysis of yield stability of barley (Hordeum vulgare L.) genotypes- using additive main effects and multiplicative interaction (AMMI) model. Iranian Journal of Crop Sciences, 9: 1-13 (In Persian).

11. Payne, R.W. 2009. "Genstat". Wiley Interdisciplinary Reviews: Computational Statistics. 1 (2): $255-$ 258

12. Samonte, S.O.PB., L.T. Wilson, A.M. McClung and J.C. Medley. 2005. Targeting cultivars onto rice growing environments using AMMI and SREG GGE biplot analyses. Crop Science, 45: 2414-2424.

13. Yan, W. and M.S. Kang. 2003. GGE Biplot Analysis: A Graphical Tool for Breeders, Geneticists, and Agronomists. CRC Press, Boca Raton, FL, USA, 269 pp.

14. Yan, W. and I. Rajcan. 2002. Biplot analysis of test sites and trait relations of soybean in Ontario. Crop Science, 42: 11-20.

15. Yan, W., M.S. Kang, B.M.S. Woods and P.L. Cornelius. 2007. GGE Biplot vs. AMMI Analysis of Genotype-by-Environment Data. Crop Science Society of America, 47: 641-653.

16. Zobel, R.W., M.J. Wright and H.G. Gauch. 1988. Statistical analysis of a yield trial. Agronomy, 80: 388-393. 


\title{
Evaluation of Genotype $\times$ Interaction Effects on Grain Yield of Barely Genotypes using Additive Main Effects and Multiplicative Interactions (AMMI)
}

\section{Hossein Ali Fallahi ${ }^{1}$, Shapour Ebrahimnejad ${ }^{2}$, Hossein Sabouri ${ }^{3}$, Iraj Lakzadeh ${ }^{4}$, Marefat Ghasemi $^{5}$ and Shirali Kohkan ${ }^{6}$}

1- Assistant Professor, Horticulture Crops Research Department, Mazandaran Agricultural and Natural Resources Research and Education Center, AREEO, Sari

2- Research Instructor, Horticulture Crops Research Department, Mazandaran Agricultural and Natural Resources

Research and Education Center, AREEO, Sari, (Corresponding author: ebrahimnejad1344@ yahoo.com) 3- Associate Professor, Gonbad-e Qabus University

4- Research Instructor, Horticulture Crops Research Department, Khozestan Agricultural and Natural Resources Research and Education Center, AREEO, Ahvaz

5- Assistant Professor, Horticulture Crops Research Department, Ardabil Agricultural and Natural Resources Research and Education Center, AREEO, Ardabil

6- Research Instructor, Horticulture Crops Research Department, Sistan and Balochestan Agricultural and Natural Resources Research and Education Center, AREEO, Zabol

Received: September 20, 2015

Accepted: April 9, 2016

\begin{abstract}
For assessment of genotype $\times$ environment $(\mathrm{G} \times \mathrm{E})$ interaction effects and determination of the most sustainable genotypes 20 barely lines were analyzed in at eight different experimental field stations. The experiments were performed based on Randomized Complete Block Design (RCBD) with three replicates. Grain yield was obtained and the results of the combined analysis of variance revealed genotype, environment and genotype $\times$ environment interaction effects were highly significant. Genotypes yield means showed that the G1, G13 and G4 genotypes had the highest grain yield. AMMI model analysis was used to determine the magnitude and significance of the genotype $\times$ environments interactions and divided G×E interaction to two principle components that explained $46 / 48 \%$ and $19 / 52 \%$ of interaction effects respectively and residuals. Based on biplot and AMMI analysis G, G genotypes were selected as candidate's lines with both best performance and grain yield sustainability. The environment E5 with high IPCA 1 and low IPCA 2 had the highest effect on determination of genotypes. The genotype G20 was selected as candidate line with high grain yield performance and stability.
\end{abstract}

Keywords: AMMI Analysis, Barely, Grain Yield, Stability 